\title{
Synthesis and Properties of Benzofuran-Fused Silole and Germole Derivatives: Reversible Dimerization and Crystal Structures of Monomers and Dimers
}

Fei-Bao Zhang, Yohei Adachi, Yousuke Ooyama, Joji Ohshita*

Department of Applied Chemistry, Graduate School of Engineering, Hiroshima University, Higashi-Hiroshima 739-8527, Japan

${ }^{1} \mathrm{H}$ and ${ }^{13} \mathrm{C}\left\{{ }^{1} \mathrm{H}\right\}$ NMR Spectra of BBFS1, BBFG1, and BBFG2 p S2

CVs of BBFS1, BBFG1, and BBFG2 p S5

$\begin{array}{ll}\text { TG of BBFS1, BBFG1, and BBFG2 } & \text { p S7 }\end{array}$

Crystal structures with atom numbering, and selected bond

distances and angles of monomers and dimers of BBFS1 and BBFG1 p S8

Packing structures of BBFS1 and BBFG1 dimers p S13

HOMO and LUMO Profiles of BBFS1, BBFG1, and BBFG2 p S14 
${ }^{1} \mathrm{H}$ and ${ }^{13} \mathrm{C}$ NMR Spectra of BBFS1, BBFG1, and BBFG2

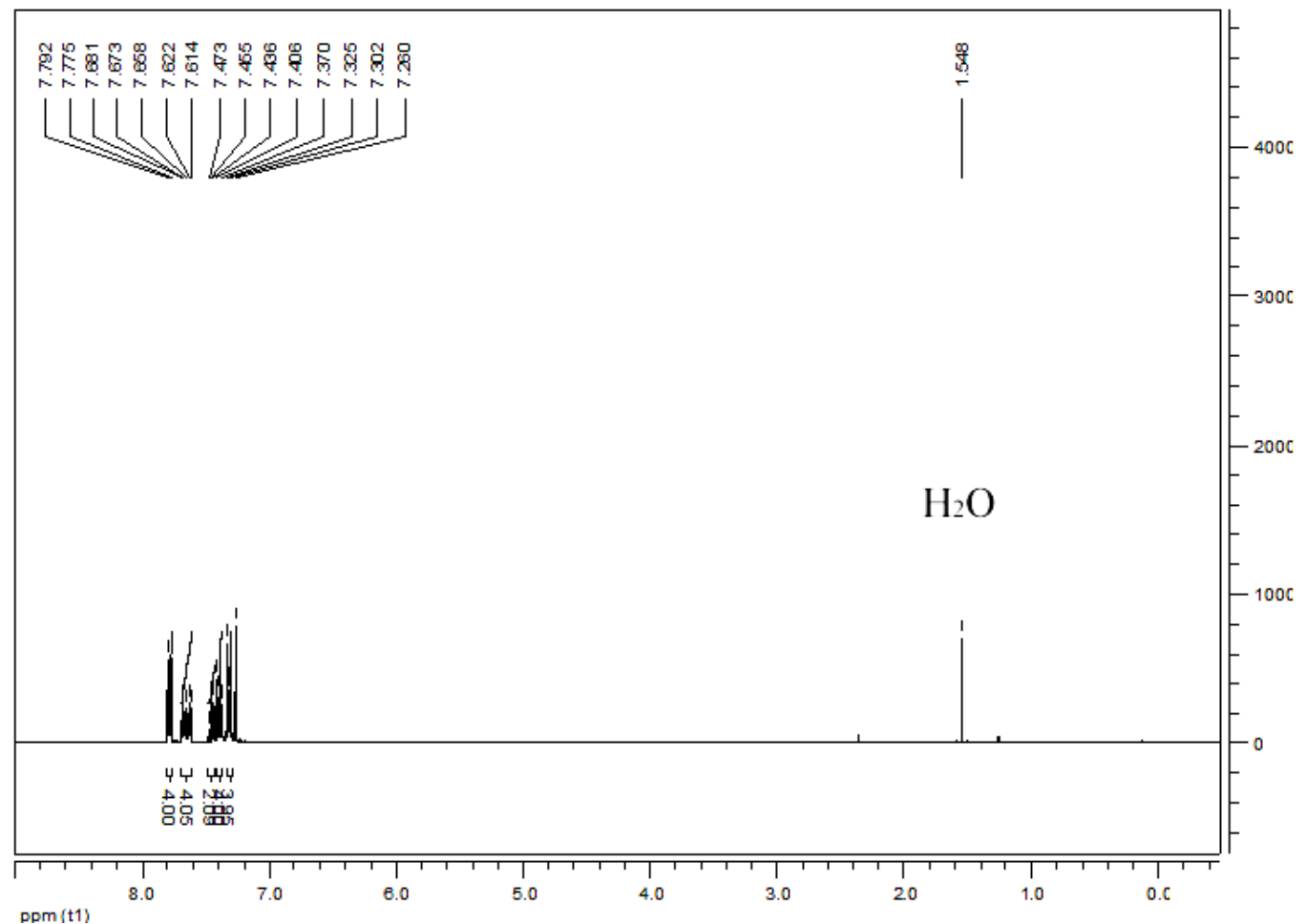

Figure S1. ${ }^{1} \mathrm{H}$ NMR spectrum of BBFS1 in $\mathrm{CDCl}_{3}$.

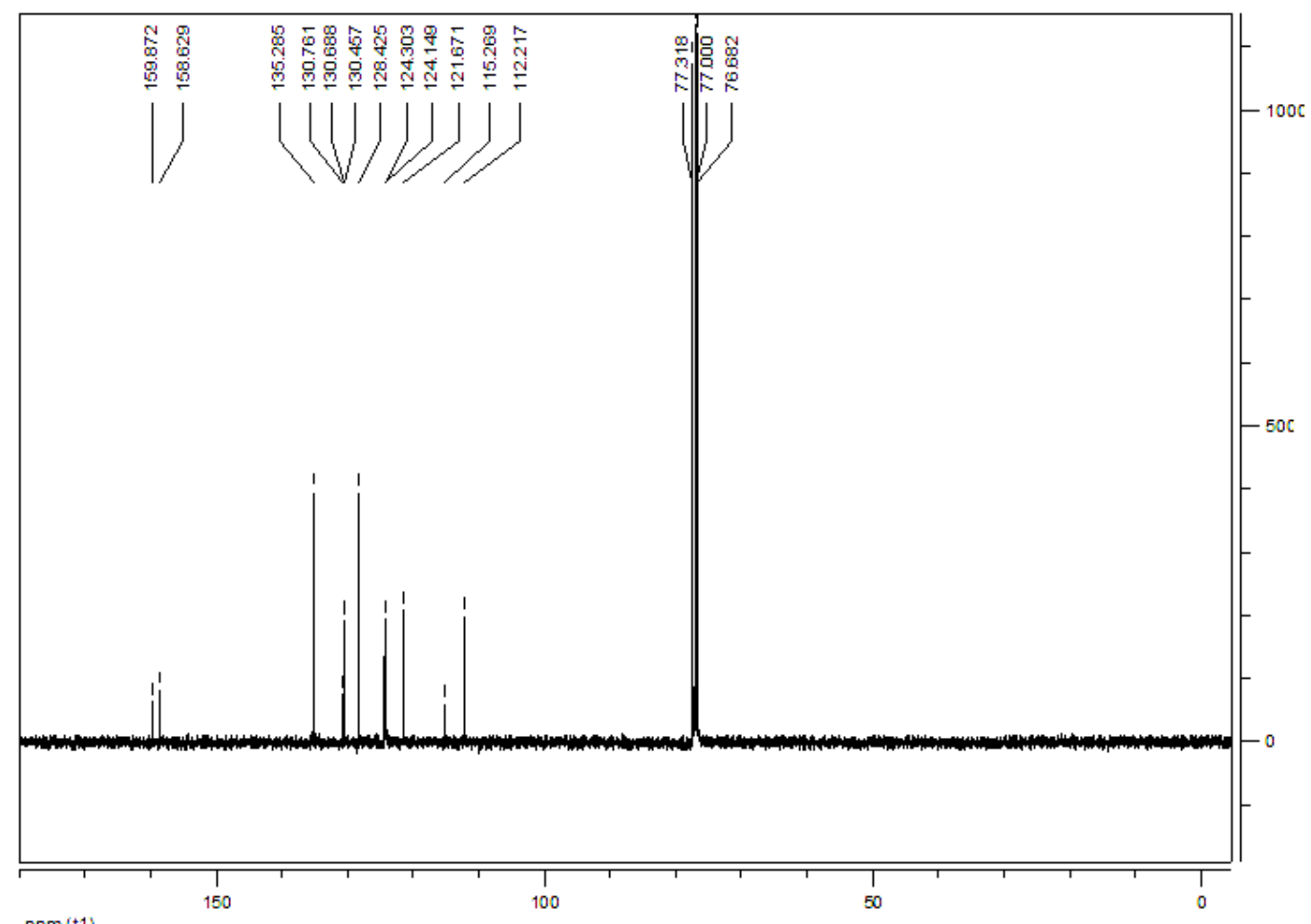

Figure S2. ${ }^{13} \mathrm{C}\left\{{ }^{1} \mathrm{H}\right\}$ NMR spectrum of BBFS1 in $\mathrm{CDCl}_{3}$. 


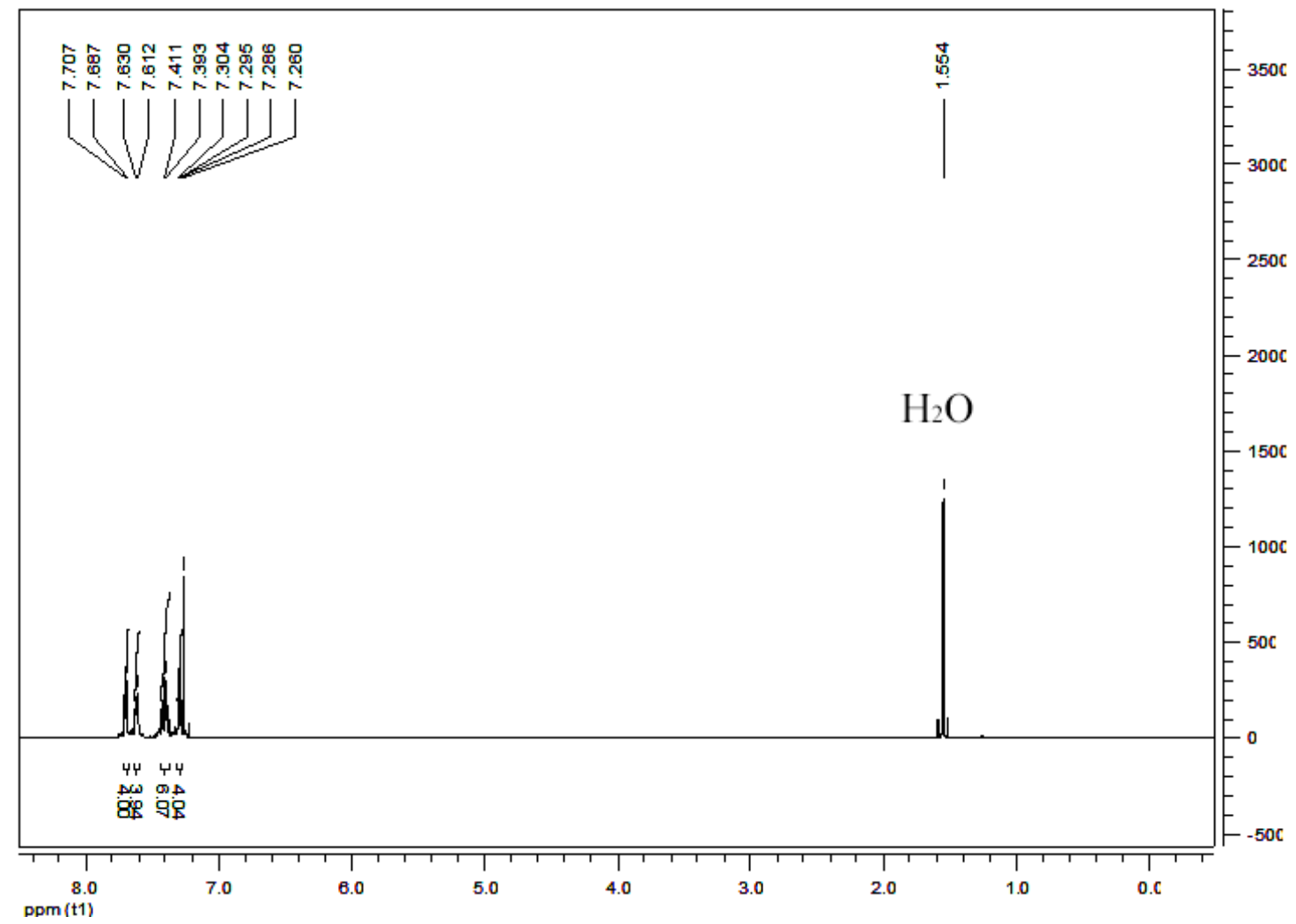

Figure S3. ${ }^{1} \mathrm{H}$ NMR spectrum of BBFG1 in $\mathrm{CDCl}_{3}$.

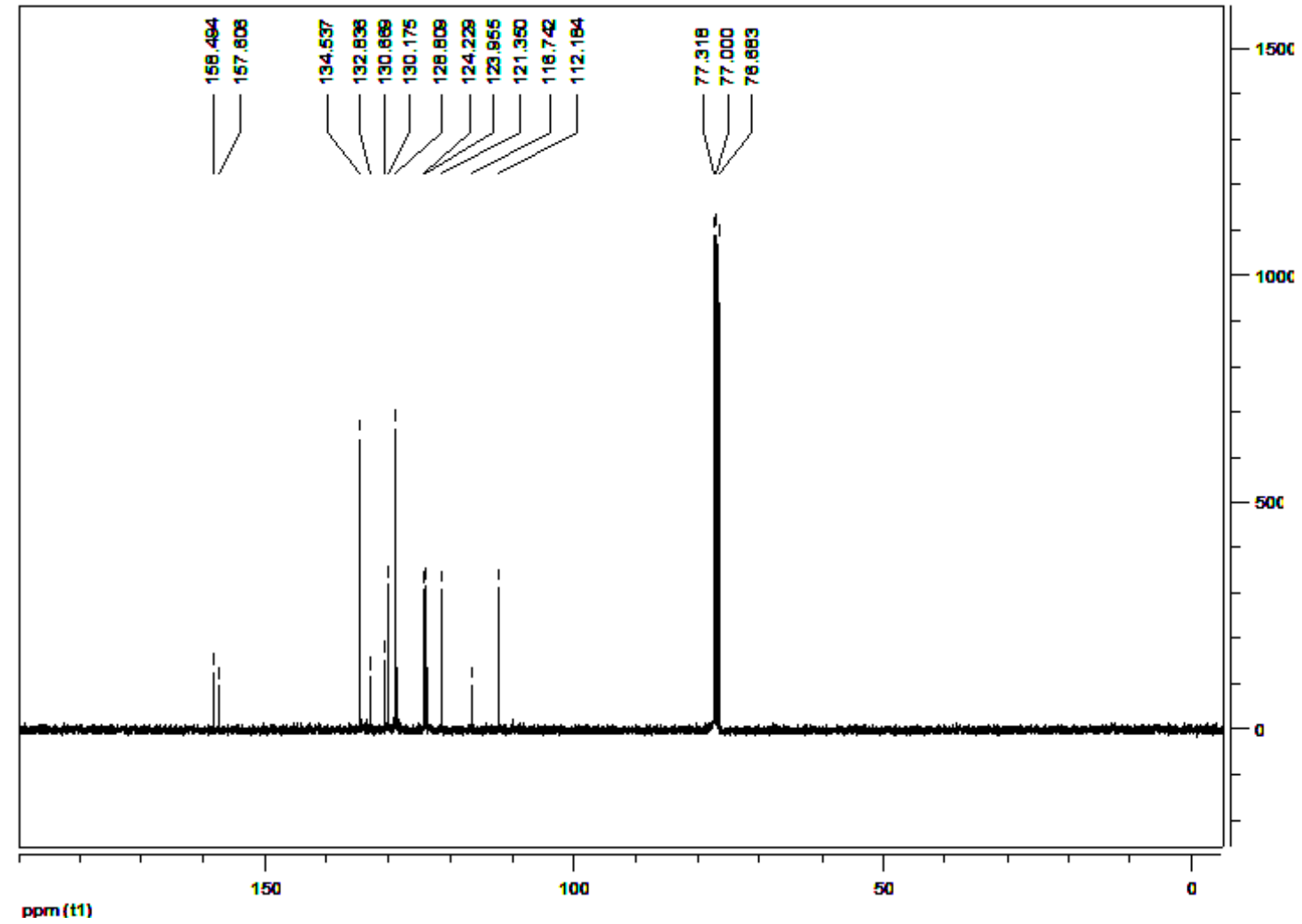

Figure S4. ${ }^{13} \mathrm{C}\left\{{ }^{1} \mathrm{H}\right\}$ NMR spectrum of BBFG1 in $\mathrm{CDCl}_{3}$. 


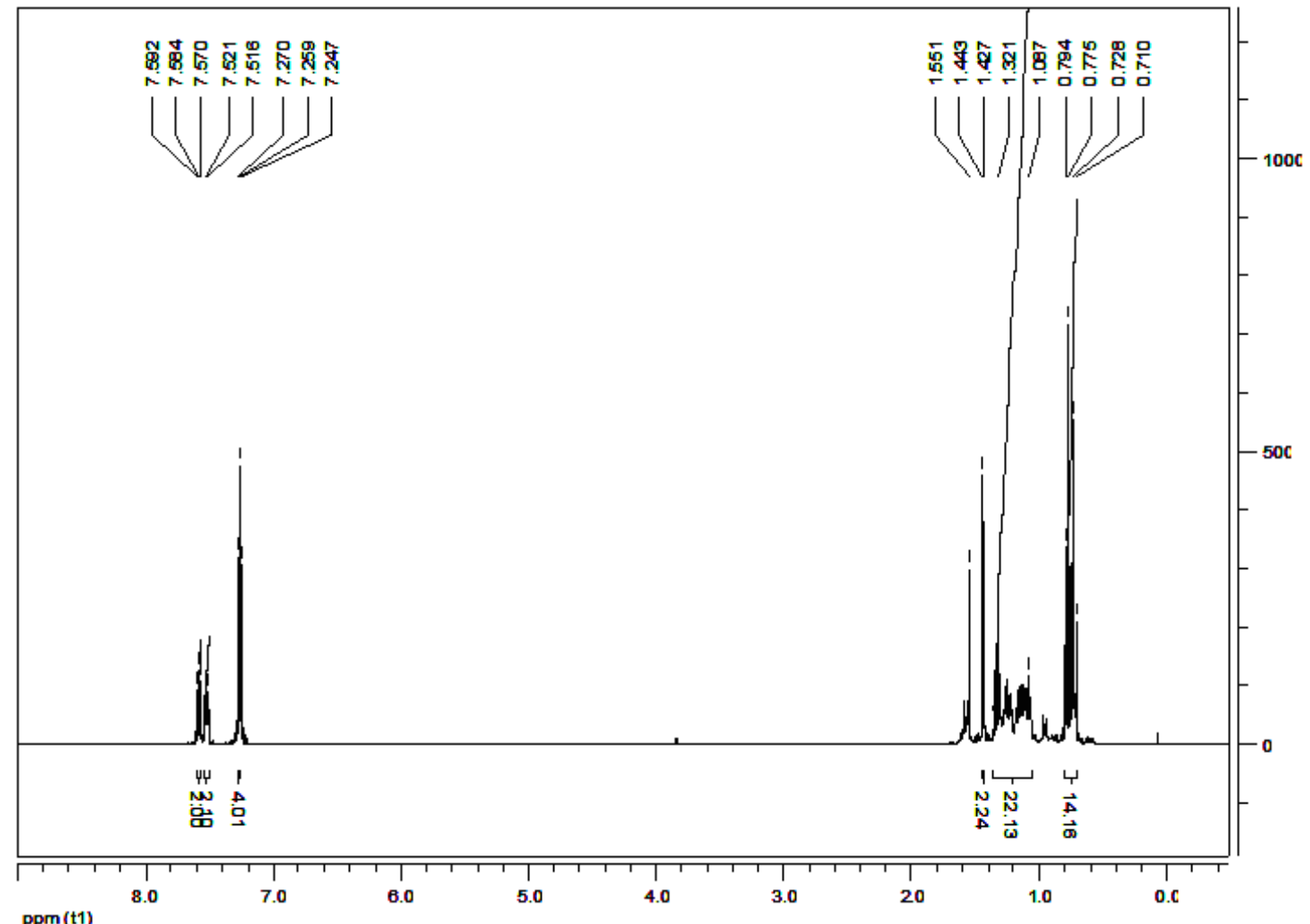

Figure S5. ${ }^{1} \mathrm{H}$ NMR spectrum of BBFG2 in $\mathrm{CDCl}_{3}$.

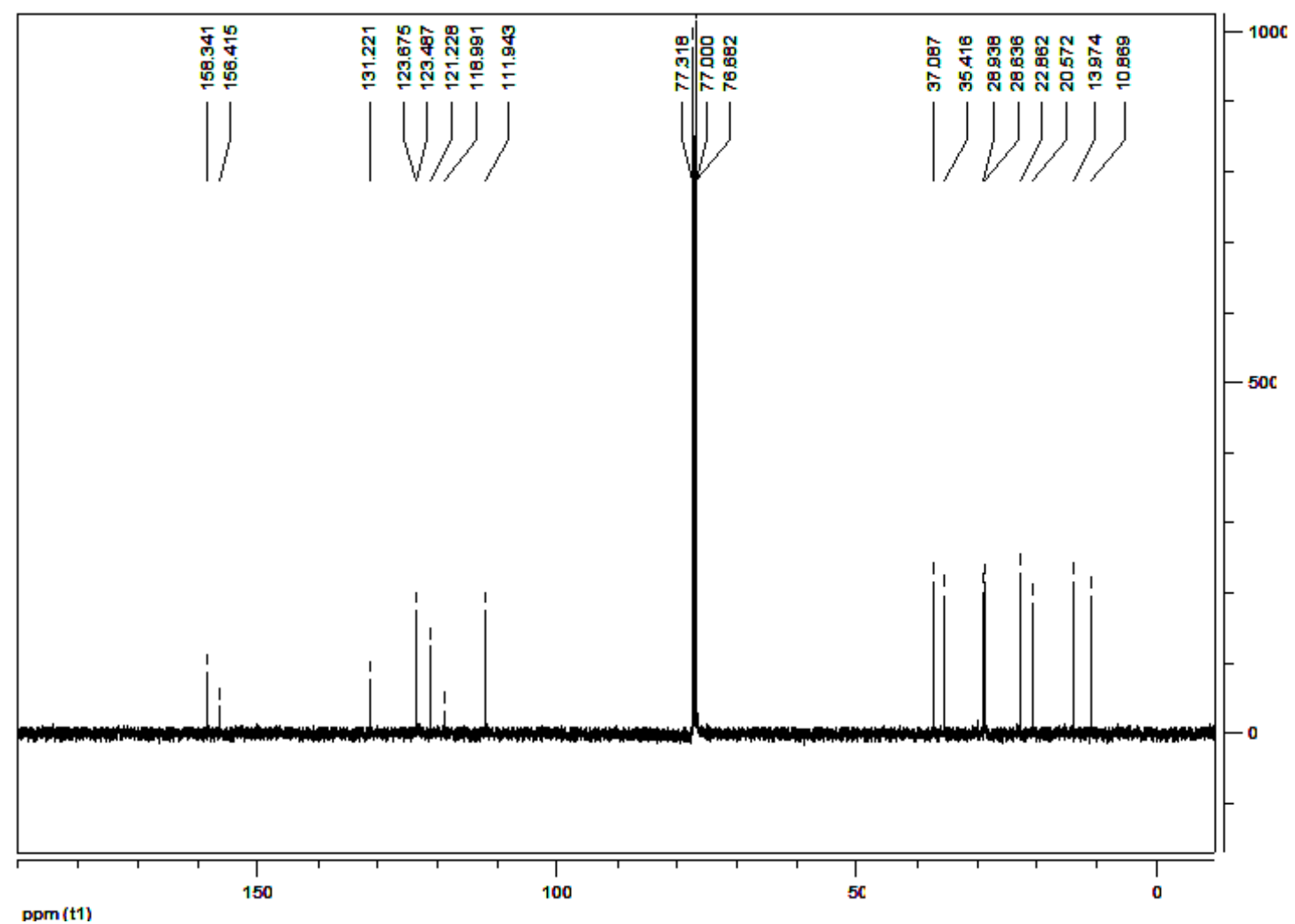

Figure S6. ${ }^{13} \mathrm{C}\left\{{ }^{1} \mathrm{H}\right\}$ NMR spectrum of BBFG2 in $\mathrm{CDCl}_{3}$. 


\section{CVS of BBFS1, BBFG1, and BBFG2}

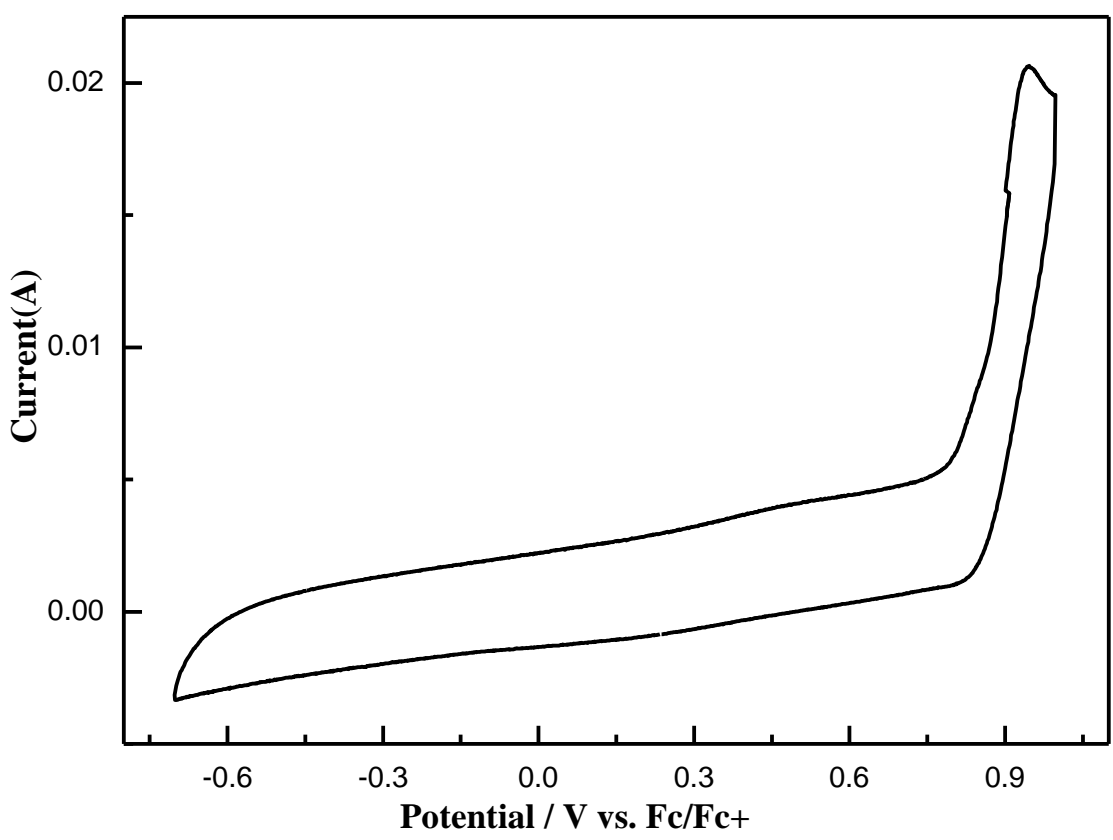

Figure S7. CVs of BBFS1 ( $2 \mathrm{mM}$ in MeCN containing $100 \mathrm{mM}$ tetrabutylammonium perchlorate as a supporting electrolyte).

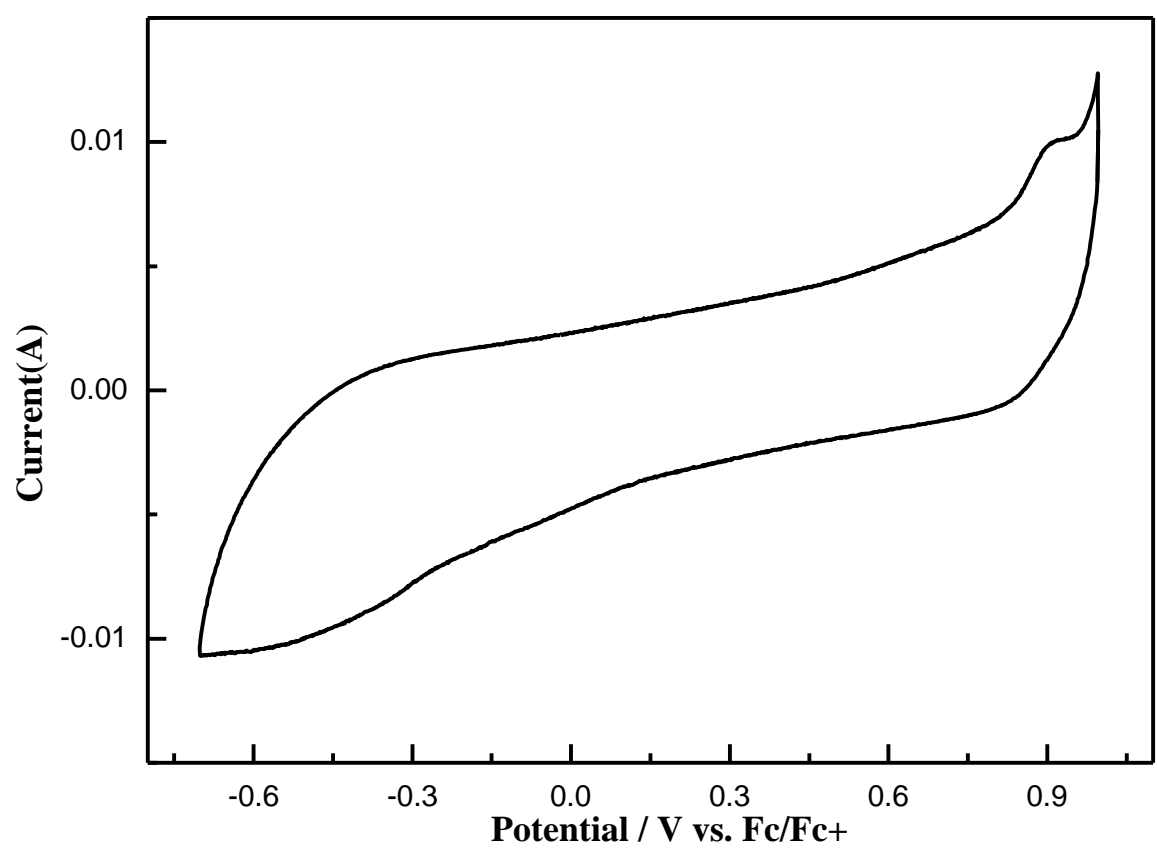

Figure S8. CVs of BBFG1 (2 mM in MeCN containing $100 \mathrm{mM}$ tetrabutylammonium perchlorate as a supporting electrolyte). 


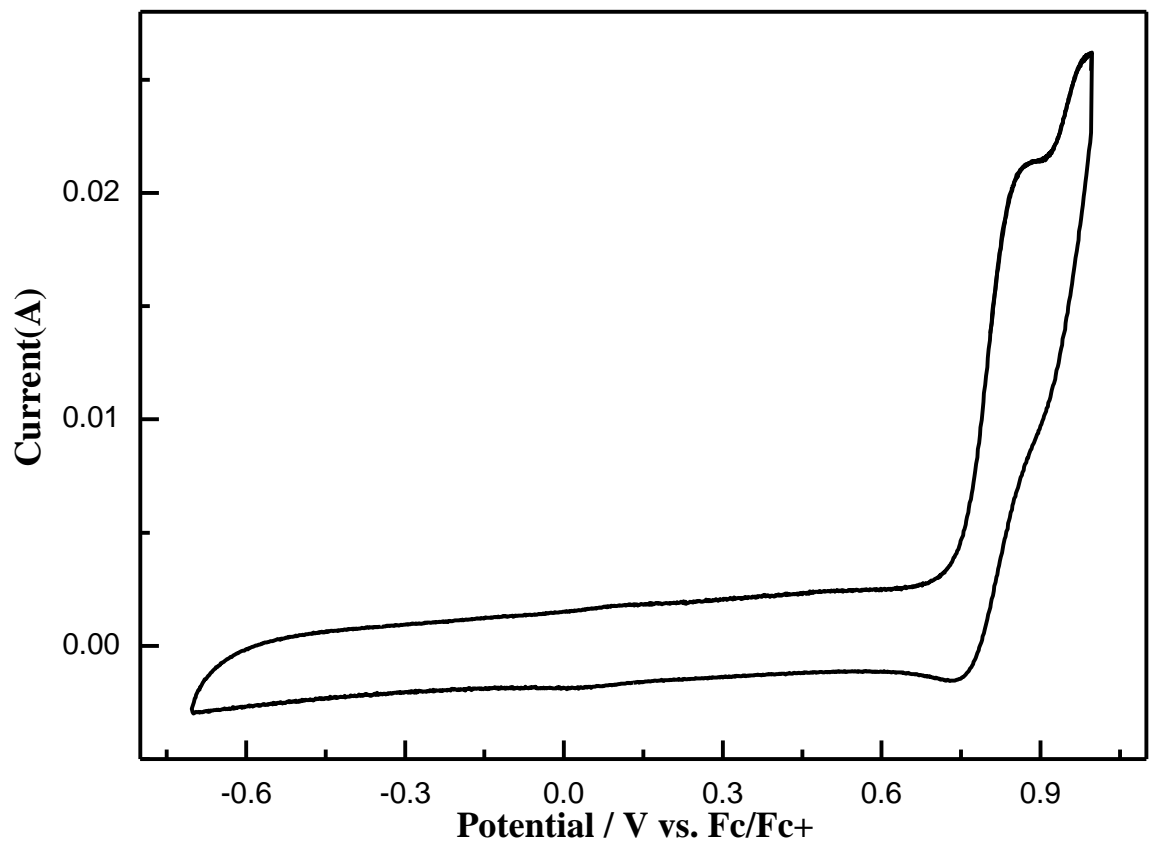

Figure S9. CVs of BBFG2 (2 mM in MeCN containing $100 \mathrm{mM}$ tetrabutylammonium perchlorate as a supporting electrolyte). 
TG OF BBFS1, BBFG1 AND BBFG2

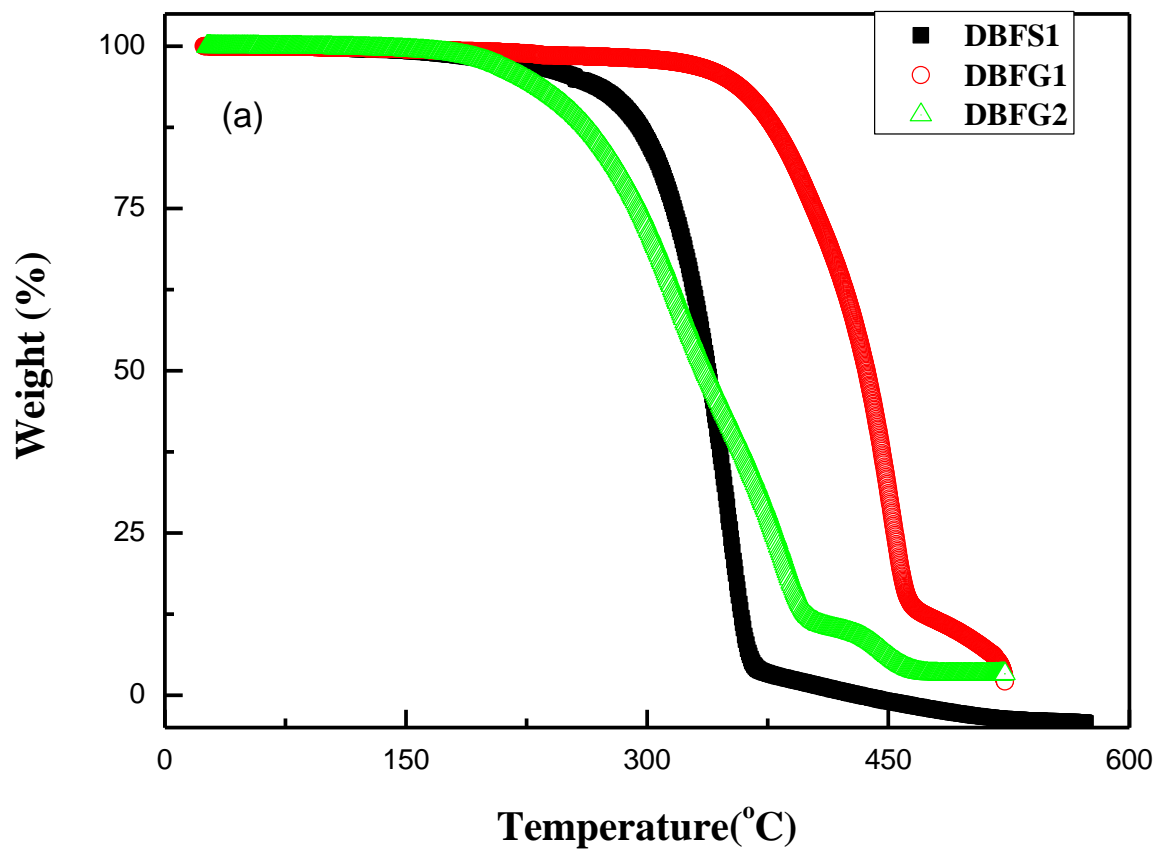

Figure S10. TG curves of BBFS1, BBFG1 and BBFG2 in nitrogen at a heating rate of $10^{\circ} \mathrm{C} / \mathrm{min}$. 
Crystal structures, and selected bond distances and angles of monomers and dimers of BBFS1 and BBFG1

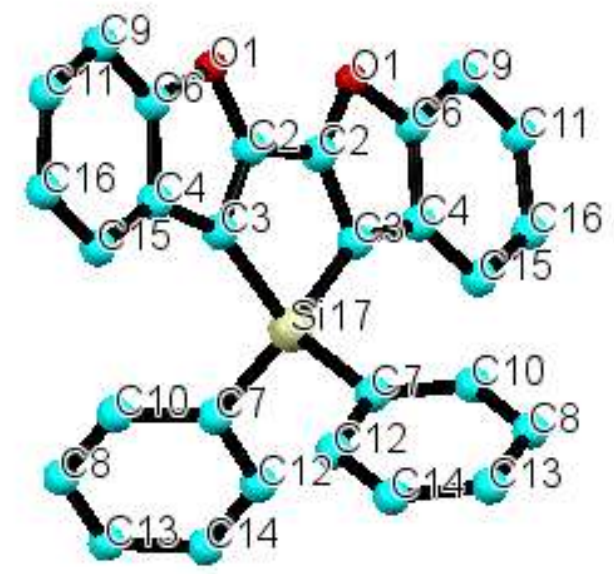

Figure S11. Ortep drawings of BBFS1 monomer with atom numbering.

Table S1. Selected Bond Distances and Angles of BBFS1

\begin{tabular}{|c|c|c|c|}
\hline \multicolumn{2}{|l|}{ Bond distance/Å } & \multicolumn{2}{|l|}{ Bond angle/deg } \\
\hline $\mathrm{C}$ (metallole)-M & $1.869(\mathrm{C} 3-\mathrm{Si} 17)$ & Inner ring C-M-C & 91.6(C3-Si17-C3) \\
\hline $\mathrm{C}(\mathrm{Ph})-\mathrm{M}$ & 1.854(C7-Si17) & $\mathrm{C}(\mathrm{Ph})-\mathrm{M}-\mathrm{C}(\mathrm{Ph})$ & $110.8(\mathrm{C} 7-\mathrm{Si} 17-\mathrm{C} 7)$ \\
\hline C-C(silole $)$ & $1.344(\mathrm{C} 3-\mathrm{C} 2)$ & C-C-M(silole) & 106.4(C2-C3-Si17) \\
\hline C-C(silole $)$ & $1.428(\mathrm{C} 2-\mathrm{C} 2)$ & C-C-C(silole $)$ & 117.7(C3-C3-C2) \\
\hline
\end{tabular}




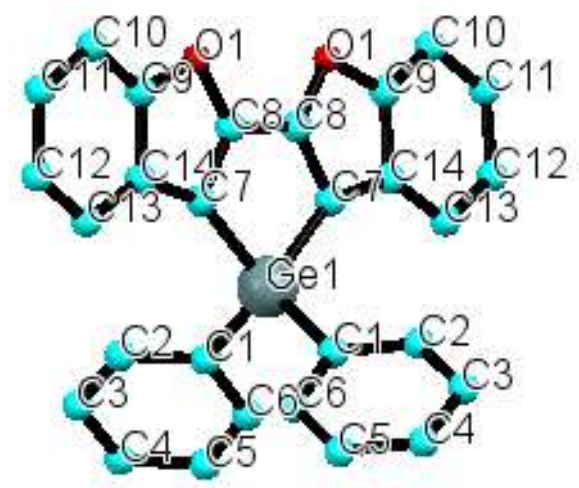

Figure S12. Ortep drawings of BBFG1 monomer with atom numbering.

Table S2. Selected Bond Distances and Angles of BBFG1

\begin{tabular}{|l|l|l|l|}
\hline \multicolumn{2}{|l|}{ Bond distance/A } & \multicolumn{2}{l|}{ Bond angle/deg } \\
\hline C (metallole)-M & $1.957(\mathrm{C} 7-\mathrm{Ge} 1)$ & Inner ring C-M-C & $89.5(\mathrm{C} 7-\mathrm{Ge} 1-\mathrm{C} 7)$ \\
\hline $\mathrm{C}(\mathrm{Ph})-\mathrm{M}$ & $1.935(\mathrm{C} 1-\mathrm{Ge} 1)$ & $\mathrm{C}(\mathrm{Ph})-\mathrm{M}-\mathrm{C}(\mathrm{Ph})$ & $110.6(\mathrm{C} 1-\mathrm{Ge} 1-\mathrm{C} 1)$ \\
\hline $\mathrm{C}-\mathrm{C}($ germole $)$ & $1.355(\mathrm{C} 7-\mathrm{C} 8)$ & C-C-M(germole $)$ & $105.9(\mathrm{C} 8-\mathrm{C} 7-\mathrm{Ge} 1)$ \\
\hline C-C(germole) & $1.431(\mathrm{C} 8-\mathrm{C} 8)$ & C-C-C(germole) & $119.2(\mathrm{C} 7-\mathrm{C} 8-\mathrm{C} 8)$ \\
\hline
\end{tabular}




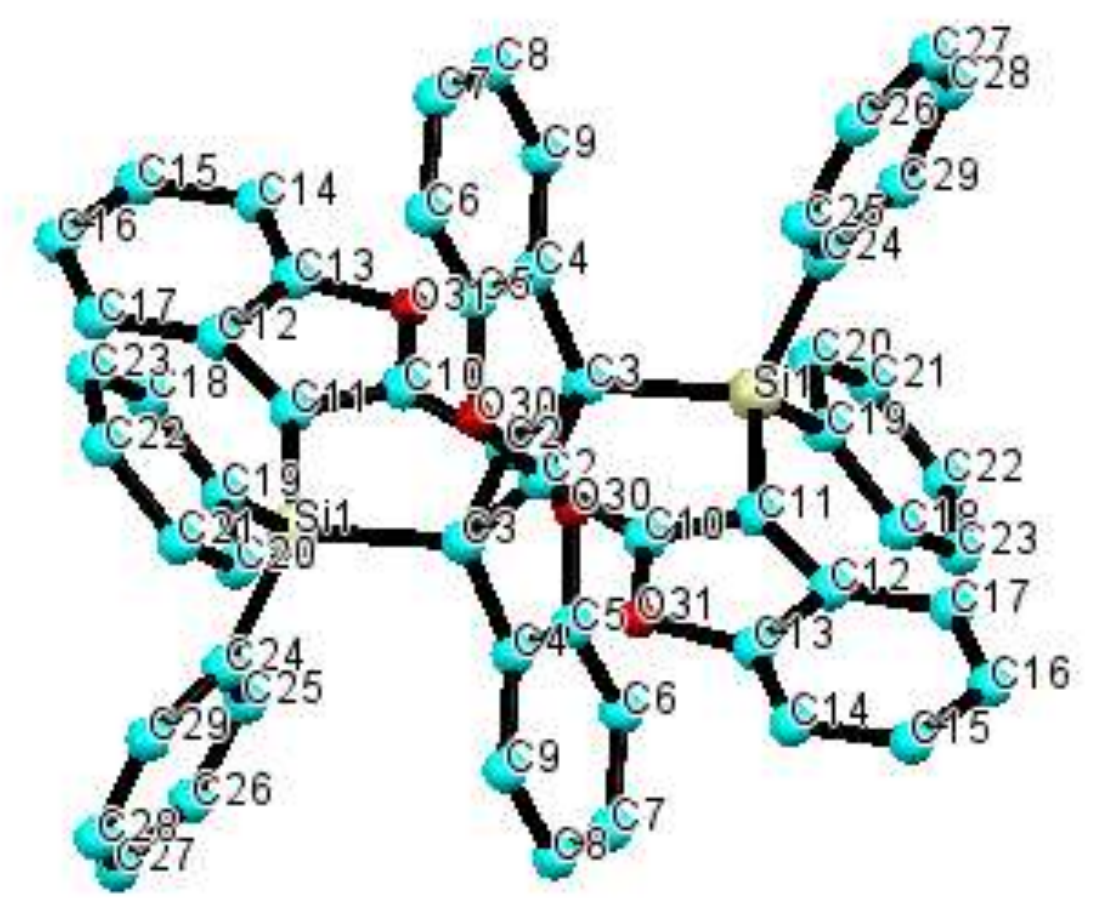

Figure S13. Ortep drawings of BBFS1 dimer with atom numbering.

Table S3. Selected Bond Distances and Angles of BBFS1 dimers

\begin{tabular}{|c|c|c|c|}
\hline \multicolumn{2}{|l|}{ Bond distance/Å } & \multicolumn{2}{|l|}{ Bond angle/deg } \\
\hline $\mathrm{C}$ (metallole)-M & $\begin{array}{l}1.856(\mathrm{C} 11-\mathrm{Si} 1) \\
1.937(\mathrm{C} 3-\mathrm{Si} 1)\end{array}$ & Inner ring C-M-C & 92.3(C3-Si1-C11) \\
\hline $\mathrm{C}(\mathrm{Ph})-\mathrm{M}$ & $\begin{array}{l}1.935(\mathrm{C} 19-\mathrm{Si} 1) \\
1.871(\mathrm{C} 24-\mathrm{Si} 1)\end{array}$ & $\mathrm{C}(\mathrm{Ph})-\mathrm{M}-\mathrm{C}(\mathrm{Ph})$ & 109.6(C19-Si1-C24) \\
\hline C-C(silole) & $\begin{array}{l}1.358(\mathrm{C} 11-\mathrm{C} 10) \\
1.477(\mathrm{C} 10-\mathrm{C} 2) \\
1.568(\mathrm{C} 2-\mathrm{C} 3)\end{array}$ & C-C-M(silole) & $\begin{array}{l}\text { 109.9(C10-C11-Si1) } \\
\text { 121.1(C2-C3-Si1) }\end{array}$ \\
\hline \multirow[t]{2}{*}{$\begin{array}{l}\text { C-C } \\
\text { (four membered ring) }\end{array}$} & $1.584\left(\mathrm{C} 2-\mathrm{C} 3{ }^{\prime}\right)$ & C-C-C(silole) & $\begin{array}{l}122.3(\mathrm{C} 11-\mathrm{C} 10-\mathrm{C} 2) \\
108.7(\mathrm{C} 10-\mathrm{C} 2-\mathrm{C} 3)\end{array}$ \\
\hline & & $\begin{array}{l}\text { C-C-C } \\
\text { (four membered ring) }\end{array}$ & $\begin{array}{l}\text { 91.3(C3-C2-C3') } \\
88.6\left(\mathrm{C} 2-\mathrm{C} 3-\mathrm{C} 2{ }^{\prime}\right)\end{array}$ \\
\hline
\end{tabular}




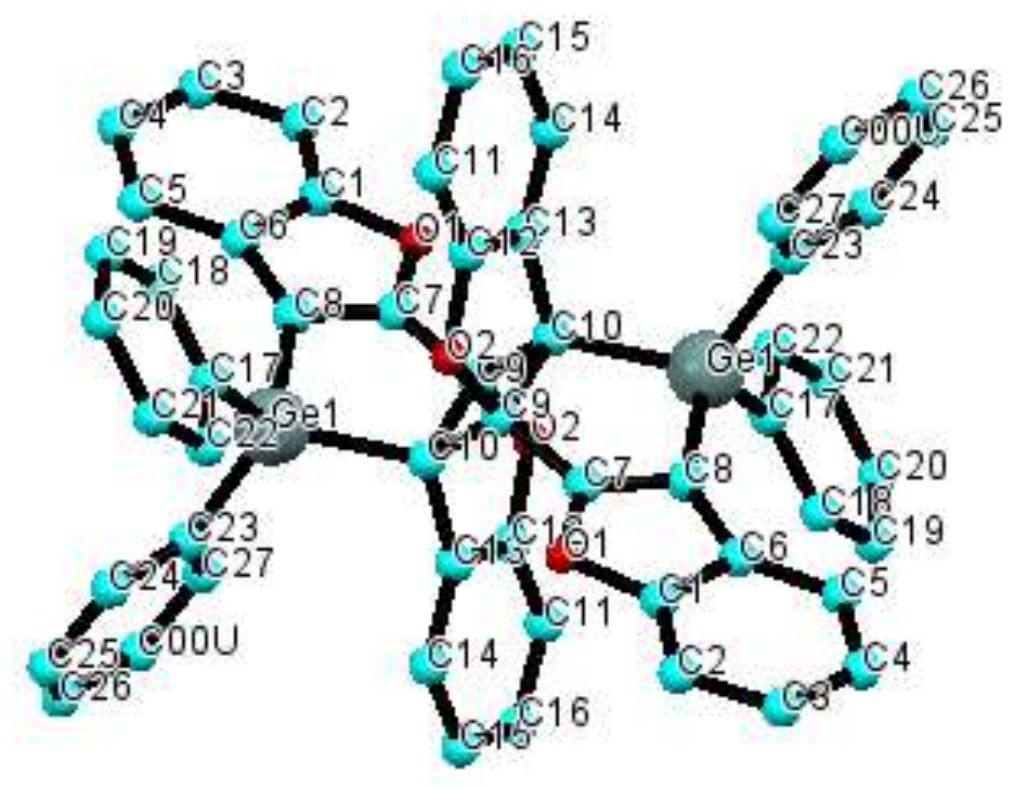

Figure S14. Ortep drawings of BBFG1 dimer with atom numbering.

Table S4. Selected Bond Distances and Angles of BBFG1 dimers

\begin{tabular}{|c|c|c|c|}
\hline \multicolumn{2}{|l|}{ Bond distance $/ \AA$} & \multicolumn{2}{|l|}{ Bond angle/deg } \\
\hline $\mathrm{C}$ (metallole)-M & $\begin{array}{l}1.934(\mathrm{C} 8-\mathrm{Ge} 1) \\
2.008(\mathrm{C} 10-\mathrm{Ge} 1)\end{array}$ & Inner ring $\mathrm{C}-\mathrm{M}-\mathrm{C}$ & 89.9(C8-Ge1-C10) \\
\hline $\mathrm{C}(\mathrm{Ph})-\mathrm{M}$ & $\begin{array}{l}1.933(\mathrm{C} 17-\mathrm{Ge} 1) \\
1.947(\mathrm{C} 23-\mathrm{Ge} 1)\end{array}$ & $\mathrm{C}(\mathrm{Ph})-\mathrm{M}-\mathrm{C}(\mathrm{Ph})$ & 109.9(C17-Ge1-C23) \\
\hline C-C(germole) & $\begin{array}{l}1.354(\mathrm{C} 7-\mathrm{C} 8) \\
1.479(\mathrm{C} 7-\mathrm{C} 9) \\
1.566(\mathrm{C} 9-\mathrm{C} 10)\end{array}$ & C-C-M(germole) & $\begin{array}{l}\text { 109.7(C7-C8-Ge1) } \\
119.7(\mathrm{C} 9-\mathrm{C} 10-\mathrm{Ge} 1)\end{array}$ \\
\hline \multirow[t]{2}{*}{$\begin{array}{l}\text { C-C } \\
\text { (four membered ring) }\end{array}$} & \multirow[t]{2}{*}{1.571 (C9-C10') } & C-C-C(germole) & $\begin{array}{l}110.0(\mathrm{C} 10-\mathrm{C} 9-\mathrm{C} 7) \\
123.4(\mathrm{C} 8-\mathrm{C} 7-\mathrm{C} 9)\end{array}$ \\
\hline & & $\begin{array}{l}\text { C-C-C } \\
\text { (four membered ring) }\end{array}$ & $\begin{array}{l}90.9\left(\mathrm{C} 10-\mathrm{C} 9-\mathrm{C} 10^{\prime}\right) \\
89.0\left(\mathrm{C}^{-C} 10-\mathrm{C} 9^{\prime}\right)\end{array}$ \\
\hline
\end{tabular}




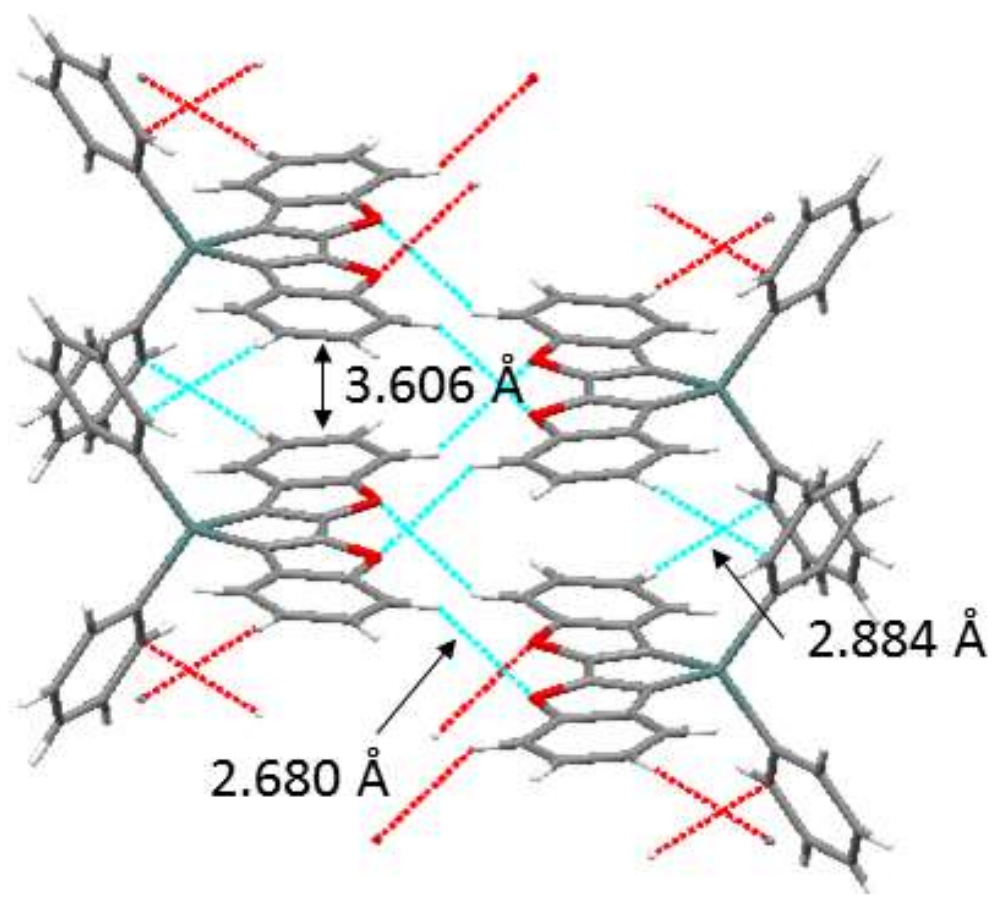

Figure S15. Solid-state intermolecular interactions of BBFG1. 


\section{Packing structures of dimers}

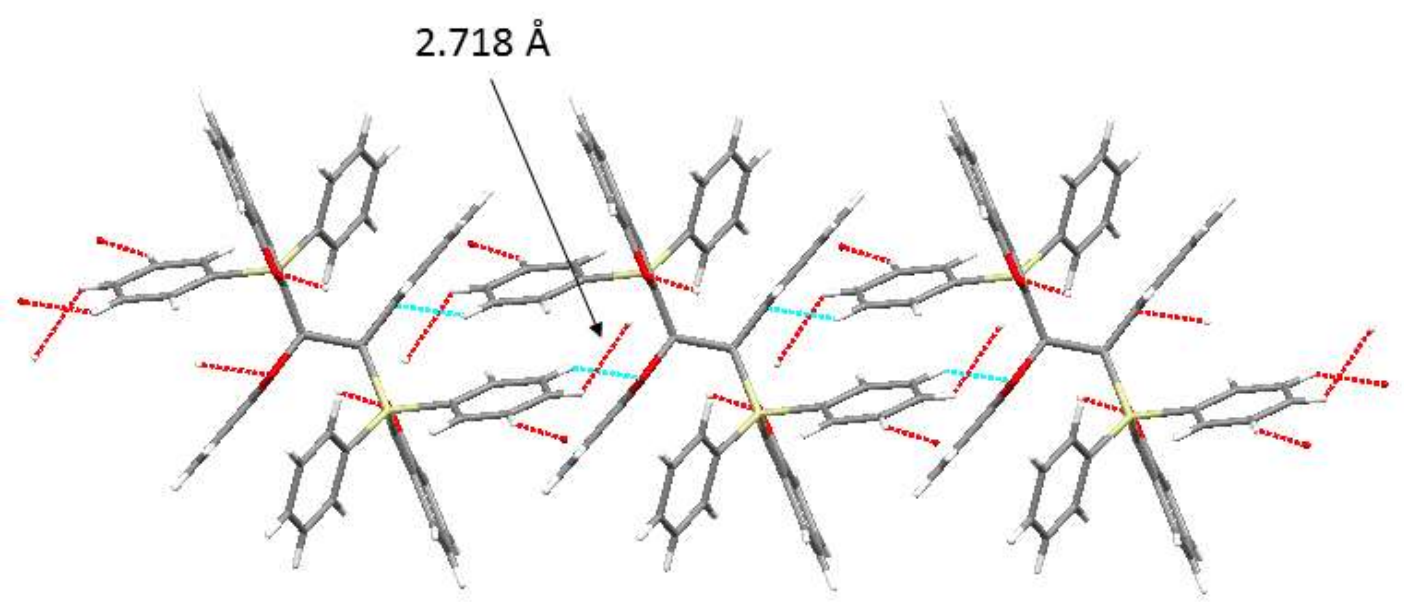

Figure S16. Solid-state intermolecular interactions of BBFS1 dimmer.

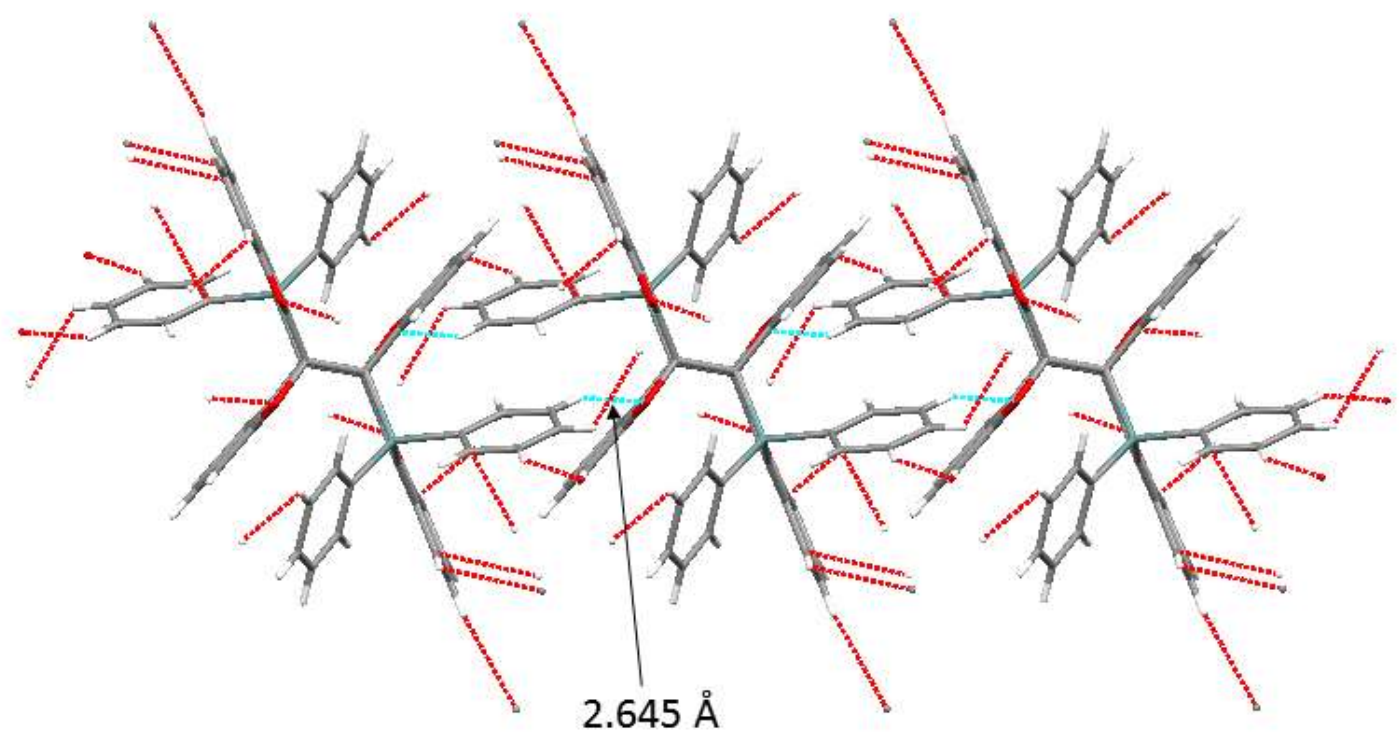

Figure S17. Solid-state intermolecular interactions of BBFG1 dimmer. 
HOMO and LUMO Profiles of BBFS1, BBFG1, and BBFG2
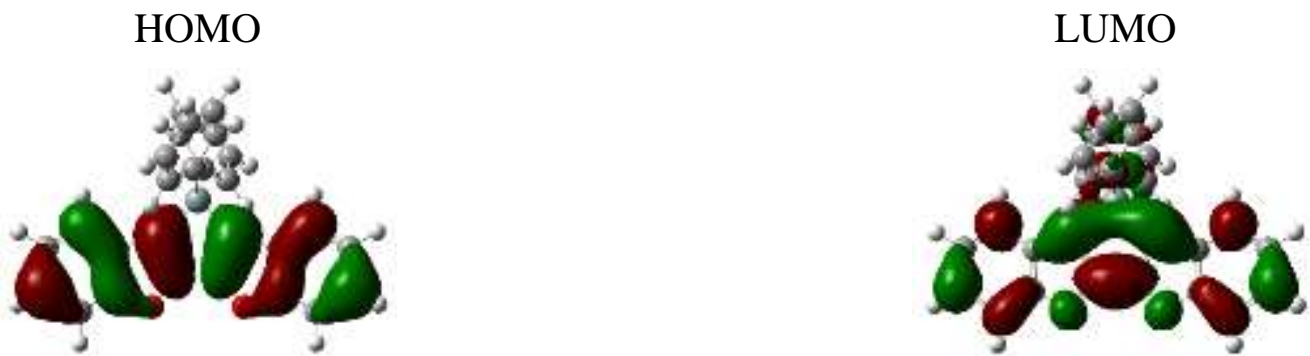

\section{BBFS1-monomer}
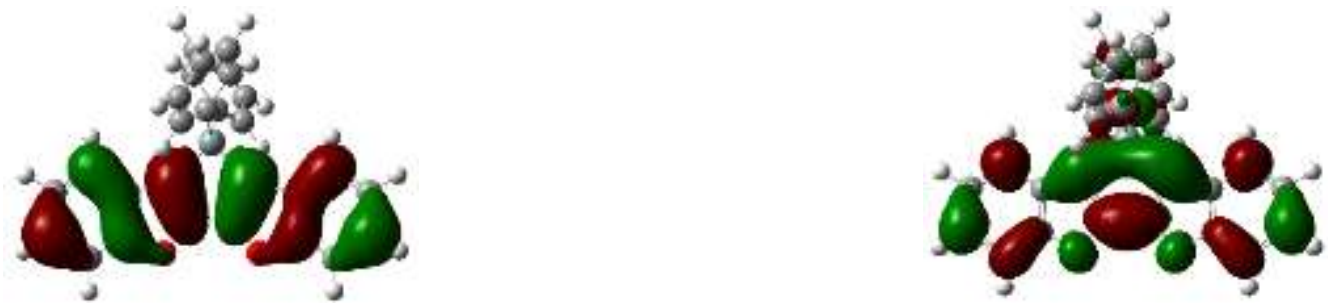

BBFG1-monomer
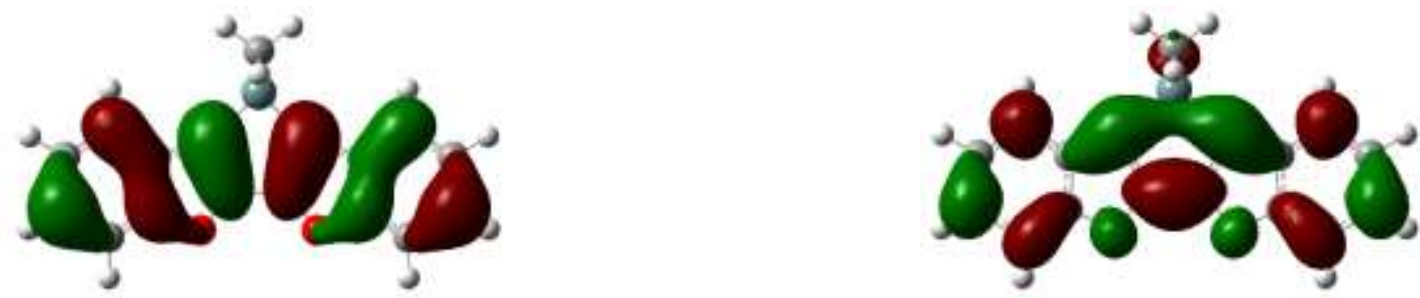

\section{BBFG3}

Figure S18. HOMO and LUMO profiles of BBFS1, BBFG1, BBFG3( 2-ethylhexyls on Ge was replaced with methyl for simplicity) monomers based on DFT calculations at the B3LYP/6-31G(d) level. 